\title{
AN ESTIMATE FOR THE GREEN'S FUNCTION
}

\author{
ALEXANDER YU. SOLYNIN
}

(Communicated by Jeremy Tyson)

In memory of Promarz M. Tamrazov, an excellent mathematician, a friend, and a wonderful person

\begin{abstract}
Let $K$ be a continuum on $\mathbb{C}$ and let $g_{\Omega(K)}(z, \infty)$ be the Green's function of $\Omega(K)=\overline{\mathbb{C}} \backslash K$. In a recent paper, V. Totik proved that $g_{\Omega(K)}\left(z_{0}, \infty\right)$ $\leq C \operatorname{dist}\left(z_{0}, \infty\right)^{1 / 2}$ with some non-sharp constant $C$ depending only on the diameter of $K$. He also used this inequality to prove new results on polynomial approximation in $\mathbb{C}$. In this note we prove a sharp version of Totik's inequality and discuss a conjectural sharp lower bound for $g_{\Omega(K)}\left(z_{0}, \infty\right)$.
\end{abstract}

Main result. Let $K$ be a continuum on the complex plane $\mathbb{C}$ and let $g_{\Omega(K)}(z, \infty)$ denote the Green's function of the unbounded component $\Omega(K)$ of $\overline{\mathbb{C}} \backslash K$ having singularity at $z=\infty$. In a recent paper [6], V. Totik used the Hölder continuity property of $g_{\Omega(K)}(z, \infty)$ to prove the absolute continuity with respect to the Lebesgue measure of some Borel measures, which in turn are found to be very useful in the theory of polynomial approximation in the complex plane. More precisely, he proved and then used the fact that for $z_{0} \in \Omega(K)$,

$$
g_{\Omega(K)}\left(z_{0}, \infty\right) \leq C \operatorname{dist}\left(z_{0}, \infty\right)^{1 / 2}
$$

with some constant $C$ depending only on the diameter of $K$.

The main goal of this paper is to prove a sharp version of inequality (1) stated in Theorem 1 It is worth mentioning that the question on the sharp lower bound for $g_{\Omega(K)}\left(z_{0}, \infty\right)$ still remains open. At the end of this note we will make some preliminary observations concerning this lower bound and discuss possible extremal configurations.

Theorem 1. Let $K$ be a continuum on $\mathbb{C}$. Then for every $z_{0} \in \Omega(K)$,

$$
g_{\Omega(K)}\left(z_{0}, \infty\right) \leq \Phi\left(\frac{\operatorname{dist}\left(z_{0}, K\right)}{\operatorname{diam}(K)}\right)<2 \frac{\operatorname{dist}\left(z_{0}, K\right)^{1 / 2}}{\operatorname{diam}(K)^{1 / 2}}
$$

where

$$
\Phi(x)=2 \log \left((1+x)^{1 / 2}+x^{1 / 2}\right) .
$$

Received by the editors August 1, 2012 and, in revised form, September 4, 2012 and September 11, 2012 .

2010 Mathematics Subject Classification. Primary 30C75, 31A15.

Key words and phrases. Green's function, extremal problem.

This research was supported by NSF grant DMS-1001882.

(C) 2014 American Mathematical Society Reverts to public domain 28 years from publication 
Furthermore, equality occurs in the first inequality in (2) if and only if $K$ is a linear segment and $z_{0}$ belongs to the line containing $K$.

The constant 2 in the second inequality in (2) is sharp.

We present two proofs of this theorem. The first one, which was suggested by the referee of the first version of this paper, shows that (2) follows from classical distortion theorems of the theory of univalent functions in the exterior of the unit disk. The second proof, which uses a different method and thus presents some interest from a methodological point of view, is based on a distortion theorem for functions univalent in an annulus proved by P. M. Tamrazov [5].

Proof 1. To prove the first inequality in (2), we may assume that $K$ does not divide the plane and that 0 lies in $K$. Then $\Omega(K)$ is the only component of $\overline{\mathbb{C}} \backslash K$. Let

$$
f(\zeta)=\operatorname{cap}(K)\left(\zeta+c_{0}+c_{-1} \zeta^{-1}+\cdots\right)
$$

map $\mathbb{D}^{*}=\{\zeta \in \overline{\mathbb{C}}:|\zeta|>1\}$ conformally onto $\Omega(K)$. Here $\operatorname{cap}(K)$ stands for the logarithmic capacity of $K$, which is defined by

$$
\operatorname{cap}(K)=\lim _{z \rightarrow \infty}\left(g_{\Omega(K)}(z, \infty)-\log |z|\right) .
$$

Notice that $f(\zeta) / \operatorname{cap}(K)=\zeta+c_{0}+c_{1} \zeta^{-1}+\cdots$ belongs to the class $\Sigma^{1}$ consisting of all normalized meromorphic and univalent in $\mathbb{D}^{*}$ functions $h(z)$ such that $0 \notin$ $h\left(\mathbb{D}^{*}\right)$. Then, by the well-known distortion theorem for this class,

$$
|\zeta|-2+|\zeta|^{-1} \leq|f(\zeta)| / \operatorname{cap}(K) \leq|\zeta|+2+|\zeta|^{-1} \text { for }|\zeta|>1
$$

see [1, p. 116, Corollary 6]. Furthermore, it is well known that

$$
2 \leq \operatorname{diam}(K) / \operatorname{cap}(K) \leq 4 ;
$$

see, for instance [3, Part Four, Chapter 2, Problem 141]. Combining the lower bound in (3) with the upper bound in (4), we get

$$
|f(\zeta)| \geq(1 / 4) \operatorname{diam}(K)\left(|\zeta|-2+|\zeta|^{-1}\right) \quad \text { for }|\zeta|>1 .
$$

To link this to the Green's function $g_{\Omega(K)}\left(z_{0}, \infty\right)$, we choose $\zeta$ such that $f(\zeta)=z_{0}$ and assume that 0 is a point of $K$ nearest to $z_{0}$. Then $\operatorname{dist}\left(z_{0}, K\right)=|f(\zeta)|$. The Green's function is conformally invariant and the Green's function of the exterior of the unit disk is given by $g_{\mathbb{D} *}(\zeta, \infty)=\log |\zeta|$. Hence, (5) is equivalent to the following:

$$
\operatorname{dist}\left(z_{0}, K\right) \geq(1 / 4) \operatorname{diam}(K)\left(e^{g_{\Omega(K)}\left(z_{0}, \infty\right)}-2+e^{-g_{\Omega(K)}\left(z_{0}, \infty\right)}\right)
$$

for $z_{0} \in \Omega(K)$. Solving (6) as a quadratic equation for $e^{g_{\Omega(K)}\left(z_{0}, \infty\right)}$ and taking into account that $g_{\Omega(K)}\left(z_{0}, \infty\right)>0$, we obtain the first inequality in (2).

Turning to the equality statement, we note that equality occurs in the first inequality in (5) if and only if $f(\zeta) / \operatorname{cap}(K)$ maps $\mathbb{D}^{*}$ conformally onto the complement of a linear segment $\left[0,4 e^{i(\alpha+\pi)}\right]$ with $\alpha=\arg f(\zeta)$; see [1, p. 116, Corollary 6]. Coincidentally, the equality in the second inequality in (6) also occurs only in the case when $K$ is a linear segment; see [3, Part Four, Chapter 2, Problem 141]. This lucky coincidence proves our statement on the cases of equality in the first relation in (2). 
To prove the second inequality in (2), we consider the function $F(x)=x^{-1 / 2} \Phi(x)$. Differentiating, we find

$$
F^{\prime}(x)=-\frac{1}{2} \frac{F_{1}(x)}{x^{5 / 2}(1+x)}
$$

where

$$
F_{1}(x)=2 \log (\sqrt{1+x}+\sqrt{x})-\frac{2}{\sqrt{x(1+x)}} .
$$

Differentiating $F_{1}(x)$ after some algebra, we find that

$$
F_{1}^{\prime}(x)=\frac{x^{1 / 2}}{(1+x)^{3 / 2}}>0 \quad \text { for } x>0 .
$$

This together with (7) shows that $F^{\prime}(x)<0$ for $x>0$. Using l'Hôpital's rule one can easily check that

$$
\lim _{x \rightarrow 0^{+}} F(x)=2, \quad \lim _{x \rightarrow \infty} F(x)=0 .
$$

Therefore, $F(x)$ strictly decreases from 2 to 0 as $x$ varies from 0 to $\infty$. In particular, this implies that $\Phi(x)<2 x^{1 / 2}$ for all $x>0$. The latter yields the second inequality in (2). In addition, the first relation in (8) shows that the factor 2 in (2) cannot be replaced by a smaller constant. The proof is complete.

Proof 2. Since $K$ is compact there are points $z_{1}, z_{2} \in K$ such that $\left|z_{1}-z_{2}\right|=$ $\operatorname{diam}(K)$. Consider a linear map $\varphi(z)=\left(z-z_{1}\right) /\left(z_{2}-z_{1}\right)$. Let $K_{\varphi}=\varphi(K)$ and $\zeta_{0}=\varphi\left(z_{0}\right)$. Then $0,1 \in K_{\varphi}, \operatorname{diam}\left(K_{\varphi}\right)=1$, and

$$
\operatorname{dist}\left(z_{0}, K\right)=\operatorname{dist}\left(\zeta_{0}, K_{\varphi}\right) \operatorname{diam}(K) .
$$

Let $\psi: \mathbb{D} \rightarrow \Omega\left(K_{\varphi}\right)$ be a Riemann mapping function from the unit disk $\mathbb{D}:=$ $\{z:|z|<1\}$ onto $\Omega\left(K_{\varphi}\right)$ such that $\psi(0)=\infty$ and $r_{0}:=\psi^{-1}\left(\zeta_{0}\right)>0$. Since the Green's function is conformally invariant we have

$$
g_{\Omega(K)}\left(z_{0}, \infty\right)=g_{\Omega\left(K_{\varphi}\right)}\left(\zeta_{0}, \infty\right)=g_{\mathbb{D}}\left(r_{0}, 0\right)=-\log r_{0} .
$$

Consider a doubly-connected domain $\mathbb{D}\left[r_{0}\right]:=\mathbb{D} \backslash\left[0, r_{0}\right], 0<r_{0}<1$. There is a unique $R, 0<R<1$, such that $\mathbb{D}\left[r_{0}\right]$ is conformally equivalent to the circular annulus $A_{R}:=\{w: R<|w|<1\}$. Let $h$ denote the conformal mapping from $A_{R}$ onto $\mathbb{D}\left[r_{0}\right]$ such that $h$ maps the unit circle $C_{1}=\{w:|w|=1\}$ onto itself. Figure 1 illustrates some notation related to the mappings and domains under consideration.

Let $f(w)=\psi(h(w))$. Then $f$ is analytic and univalent on $A_{R}$. Let $\Gamma_{0}$ and $\Gamma_{\infty}$ denote the bounded component of $\overline{\mathbb{C}} \backslash f\left(A_{R}\right)$ and the unbounded component of $\overline{\mathbb{C}} \backslash f\left(A_{R}\right)$, respectively. Then the bounded component $\Gamma_{0}$ contains the points 0,1 and the continuum $f\left(C_{1}\right)$ while the unbounded component $\Gamma_{\infty}$ contains the point $\zeta_{0}$ and the continuum $f\left(C_{R}\right)$.

The latter observation shows that the function $f\left(e^{i \alpha} z\right)$ with an appropriate $\alpha \in$ $\mathbb{R}$ belongs to the class $F$ studied by P. Tamrazov in [5]. Among other results established in [5] Tamrazov proved the following important inequality:

$$
\operatorname{dist}\left(f\left(C_{1}\right), f\left(C_{R}\right)\right) \geq t(R),
$$

where $t(R)>0$ is such that the doubly-connected domain $D(t(R))$, where $D(t):=$ $\mathbb{C} \backslash([-\infty,-t] \cup[0,1])$, is conformally equivalent to $A_{R}$. 


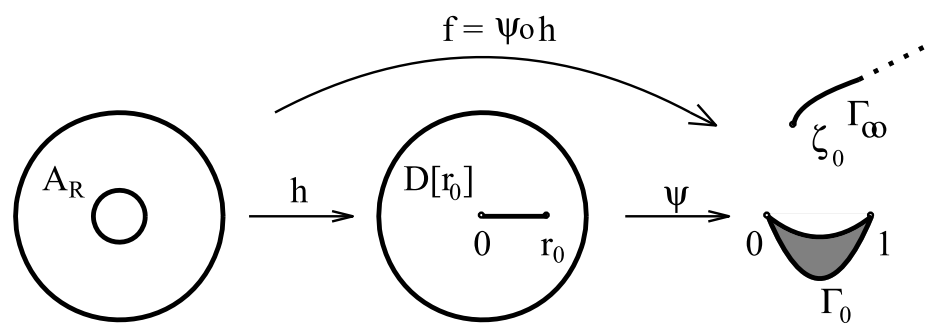

FiguRE 1. Mapping functions and domains.

Since $A_{R}$ is conformally equivalent to $\mathbb{D}\left[r_{0}\right]$ it follows that $D(t(R))$ is also conformally equivalent to $\mathbb{D}\left[r_{0}\right]$. Let $j(\tau)=-\left(\tau+\tau^{-1}-2\right) / 4$. The function $j(\tau)$ maps $\mathbb{D}\left[r_{0}\right]$ conformally and one-to-one onto the domain $D(\rho)$ with $\rho=\left(r_{0}+r_{0}^{-1}-2\right) / 4$. Since $\mathbb{D}\left[r_{0}\right]$ is conformally equivalent to $D(t(R))$ we must have $\rho=t(R)$, which implies that

$$
t=t(R)=\left(r_{0}+r_{0}^{-1}-2\right) / 4 .
$$

Solving this for $r_{0}$, we obtain

$$
r_{0}=\left((1+t)^{1 / 2}+t^{1 / 2}\right)^{-2} .
$$

It is clear that $r_{0}$ defined by (12) strictly decreases from 1 to 0 as $t$ runs from 0 to $\infty$. Since $\zeta_{0} \in f\left(C_{R}\right)$, we have

$$
\operatorname{dist}\left(\zeta_{0}, K_{\varphi}\right) \geq \operatorname{dist}\left(f\left(C_{1}\right), f\left(C_{R}\right)\right) .
$$

From (9)-(13), we conclude that

$$
g_{\Omega(K)}\left(z_{0}, \infty\right) \leq \Phi(t(R)) \leq \Phi\left(\frac{\operatorname{dist}\left(z_{0}, K\right)}{\operatorname{diam}(K)}\right) .
$$

It was shown in [5] that equality occurs in (11) if and only if $f$ maps $A_{R}$ onto the complement of the segments $[-\infty,-t(R)]$ and $[0,1]$ or it maps $A_{R}$ onto the complement of the segments $[1+t(R), \infty]$ and $[0,1]$. This together with the strict monotonicity property of the function $\Phi(x)$ for $t \geq 1$ implies the statement about the cases of equality in the first inequality in (2).

Lower bound. We stress here that inequalities (3) and (4) cannot provide a sharp lower bound for $g_{\Omega(K)}\left(z_{0}, \infty\right)$ because the sign of equality occurs in the second inequality in (3) only when $K$ is a linear segment (see [1, p. 116, Corollary 6]), while the sign of equality occurs in the first inequality in (44) only when $K$ is a disk (see [3, Part Four, Chapter 2, Problem 141]). Using (3) and (4) as in Proof 1 above, we obtain the following non-sharp lower bound:

$$
g_{\Omega(K)}\left(z_{0}, \infty\right) \geq \Phi_{1}\left(\frac{\operatorname{dist}\left(z_{0}, K\right)}{\operatorname{diam}(K)}\right) \geq \log \left(\frac{\operatorname{dist}\left(z_{0}, K\right)}{\operatorname{diam}(K)}\right)-\log 2,
$$

with $\Phi_{1}(x)=\log (x-1+\sqrt{x(x-2)})$. Of course, (14) makes sense only if $\operatorname{dist}\left(z_{0}, K\right)$ $\geq 2 \operatorname{diam}(K)$. 
Seeking a sharp lower bound for $g_{\Omega(K)}\left(z_{0}, \infty\right)$, we first note that the trivial lower bound $g_{\Omega(K)}\left(z_{0}, \infty\right)>0$ is best possible if $\operatorname{diam}(K) \geq 2 \operatorname{dist}\left(z_{0}, K\right)$. Indeed, assume without loss of generality that $z_{0}=0$ and $\operatorname{dist}\left(z_{0}, K\right)=1$. Then for $0<\varepsilon<\pi$ and $d \geq 2$ consider compact sets

$$
E(d, \varepsilon)=\left\{z=e^{i \theta}: 0 \leq \theta \leq 2 \pi-\varepsilon\right\} \cup[1, d-1] .
$$

We have $\operatorname{diam}(E(d, \varepsilon))=d$ and

$$
g_{\Omega(E(d, \varepsilon))}(0, \infty) \rightarrow 0 \quad \text { as } \varepsilon \rightarrow 0 .
$$

The latter equation follows from well-known results on convergence of sequences of Green's functions of domains convergent to a kernel; see, for instance, [6].

Suppose now that $z_{0}=0$ and $K$ is a continuum such that

$$
d:=\operatorname{diam}(K)<2 \operatorname{dist}\left(z_{0}, K\right)=2 .
$$

Then there is a half-plane $H_{0}$ such that $0 \in \partial H_{0}$ and $K \subset \overline{H_{0}}$. In what follows, we assume that $H_{0}$ is the left half-plane $\{z: \Re z<0\}$.

Let $\widehat{K}$ denote the set of constant width $d$ containing $K$; i.e. $\widehat{K}$ is the intersection of all closed disks of radius $d$ centered at the points of the convex hull of $K$. Let $\widehat{S}=\left(\widehat{K} \cap \overline{H_{0}}\right) \backslash \mathbb{D}$. Then $K \subset \widehat{S}, \operatorname{diam}(\widehat{S})=d$, $\operatorname{dist}(0, \widehat{S})=1$ and $g_{\Omega(\widehat{S})}(0, \infty) \leq$ $g_{\Omega(K)}(0, \infty)$. Therefore, when finding the sharp lower bound for $g_{\Omega(K)}(0, \infty)$, it is sufficient to work with compact sets $K$ such that $\widehat{S}=K$.

The following monotonicity property is well known. It follows, for example, from the polarization result of Theorem 1 in [4]. If $K \subset H_{0}$, then for each fixed $y \in \mathbb{R}$ the function $g_{\Omega(K)}(x+i y, \infty)$ strictly increases for $x>0$. This monotonicity property easily implies that in the problem on the sharp lower bound for $g_{\Omega(K)}\left(z_{0}, \infty\right)$ we may assume that the intersection $K \cap \mathbb{T}$ is not empty. Here $\mathbb{T}=\{z:|z|=1\}$. Let $\gamma_{K}:=K \cap \mathbb{T}=\left\{e^{i \theta}: \theta_{1} \leq \theta \leq \theta_{2}\right\}$, where $\pi / 2 \leq \theta_{1} \leq \theta_{2} \leq 3 \pi / 2$, and let $H$ be a half-plane such that $e^{i \theta_{1}}, e^{i \theta_{2}} \in \partial H$ and $\gamma_{K} \subset \bar{H}$. Since $K=\widehat{S}$, it follows that $K \subset \bar{H}$. Let $K^{\prime}=\left\{z:-e^{i \frac{\theta_{1}+\theta_{2}}{2}} z \in K\right\}$. Then $-1 \in \gamma_{K^{\prime}}$, the arc $\gamma_{K^{\prime}}$ is symmetric with respect to $\mathbb{R}$, and $g_{\Omega\left(K^{\prime}\right)}(0, \infty)=g_{\Omega(K)}(0, \infty)$.

Combining our observations, we conclude that when working on the problem on the sharp lower bound for $g_{\Omega(K)}(0, \infty)$, we may restrict ourselves to the collection of sets $\mathcal{C}_{d}$ with $0<d<2$, consisting of continua $K$ such that $\widehat{S}=K \subset H_{0}$, $\operatorname{diam}(K)=d$, and $K \cap \mathbb{T}$ contains the point $z=-1$ and is symmetric with respect to $\mathbb{R}$.

An interesting example of a compact set with all these properties, which seems to be a natural candidate for the extremal configuration in the problem under consideration, is provided by the circular triangle $Q(d) \in \mathcal{C}_{d}$ defined by

$$
Q(d):=\left\{z:|z| \geq 1,\left|z+(1 / 2)\left(\sqrt{4-d^{2}} \pm i d\right)\right| \leq d\right\}, \quad 0<d \leq 2 .
$$

Thus, $Q(d)$ is the Reulaux triangle minus a disk. As is well known, the Reulaux triangle plays the role of the extremal configuration in several problems in geometry. We conjecture that its portion $Q(d)$ defined by (16) is extremal for the problem of determining the sharp lower bound for $g_{\Omega(K)}\left(z_{0}, \infty\right)$. 


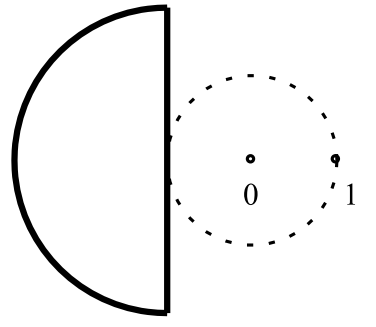

a) $\mathrm{d}=1.8, \mathrm{r}=1$

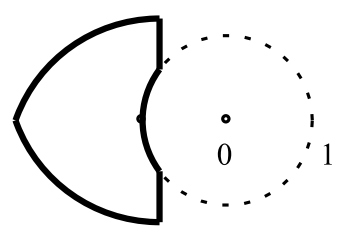

b) $\mathrm{d}=1.8, \mathrm{r}=0.4$

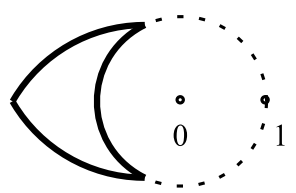

c) $\mathrm{d}=1.8, \mathrm{r}=0.22$

Figure 2. Circular polygons $Q(d, r)$.

Conjecture 1. Let $K$ be a continuum on $\mathbb{C} \backslash \mathbb{D}$ such that $\operatorname{diam}(K)=d$ with $0<d<2$. Then $0 \in \Omega(K)$ and

$$
g_{\Omega(K)}(0, \infty) \geq g_{\Omega(Q(d))}(0, \infty)
$$

with the sign of equality in (17) if and only if $K$ coincides with $Q(d)$ up to rotation about the origin.

Let $K \in \mathcal{C}_{d}, 0<d<2$, and let $r_{K}=-\max \{\Re z: z \in K\}$. Then $(1 / 2) \sqrt{4-d^{2}} \leq$ $r_{K} \leq 1$ and $\gamma_{K}$ is the arc of $\mathbb{T}$ joining the points $z=-e^{-i \arccos r_{K}}$ and $z=$ $-e^{i \arccos r_{K}}$ such that $-1 \in \gamma_{K}$. For given $d$ and $r$ such that $0<d \leq 2$, $(1 / 2) \sqrt{4-d^{2}} \leq r \leq 1$, we denote by $Q(d, r)$ the circular quadrilateral defined by

$$
Q(d, r)=\left\{z:|z| \geq 1, \Re z \leq-r,\left|z+r \pm i \sqrt{1-r^{2}}\right| \leq d\right\} .
$$

In two limit cases, when $r=1$ and $r=(1 / 2) \sqrt{4-d^{2}}, Q(d, r)$ represents simpler configurations. Precisely, $Q(d, 1)$ is the half-disk $\{z:|z+1| \leq d, \Re z \leq-1\}$, while $Q\left(d,(1 / 2) \sqrt{4-d^{2}}\right)$ coincides with the circular triangle defined by (16). Thus, $Q\left(d,(1 / 2) \sqrt{4-d^{2}}\right)=Q(d)$. These limit cases along with a typical shape of $Q(d, r)$ are shown in Figure 2. To abbreviate our notation we introduce the function $G(d, r):=g_{\Omega(Q(d, r))}(0, \infty), 0<d \leq 2,(1 / 2) \sqrt{4-d^{2}} \leq r \leq 1$. Then we put $G(d):=G\left(d,(1 / 2) \sqrt{4-d^{2}}\right)$ and $G_{1}(d):=G(d, 1)$.

If $K \in \mathcal{C}_{d}$, then one can easily show that $K \subset Q\left(d, r_{K}\right)$. Therefore,

$$
G\left(d, r_{K}\right)=g_{\Omega\left(Q\left(d, r_{K}\right)\right)}(0, \infty) \leq g_{\Omega(K)}(0, \infty) .
$$

The latter inequality implies that the circular triangle $Q(d)$ would be extremal for the problem on the sharp lower bound for $g_{\Omega(K)}(0, \infty)$ if inequality (18) of the following conjecture holds.

Conjecture 2. For a fixed $d, 0<d \leq 2$, the function $G(d, r)$ strictly increases as $r$ runs from $(1 / 2) \sqrt{4-d^{2}}$ to 1 .

In particular,

(18) $G(d, r)>G(d)$ for all $d$ and $r$ such that $0<d \leq 2,(1 / 2) \sqrt{4-d^{2}}<r \leq 1$. 
We want to stress here that the diameter of $Q(d, r)$ equals $2\left(d-\sqrt{1-r_{K}^{2}}\right)$ and is strictly greater than $d$ if $r_{K}<(1 / 2) \sqrt{4-d^{2}}$. Therefore, $Q\left(d, r_{K}\right)$ does not belong to the class $\mathcal{C}_{d}$ in this case. Thus, Conjecture 2 suggests that inequality (17) holds for a broader class of continua than it is described in Conjecture 1.

Finding a closed form for the function $G(d, r)$ will involve finding a conformal mapping from the upper plane $\mathbb{H}=\{z: \Im z>0\}$ onto the complement $\Omega(Q(d, r))$ of the circular quadrilateral $Q(d, r)$. As is well known (see, for instance, [2, Section V.7]), the Schwarz equation for such mapping contains one accessory parameter, which can be found explicitly only in rare cases, and the case of the quadrilateral $Q(d, r)$ is not among those if $(1 / 2) \sqrt{4-d^{2}}<r<1$. Of course, the Green's function of the exterior of a half-disk can be easily calculated. Indeed, the function

$$
f(z)=\left(\frac{d+i(z+1)}{d-i(z+1)}\right)^{2 / 3}
$$

with the branch of the radical chosen such that

$$
f(\infty)=e^{\frac{2 \pi i}{3}} \text { and } \quad f(0)=e^{\frac{4 i}{3} \operatorname{arccot} d}
$$

maps $\Omega(Q(d, 1))$ conformally and one-to-one onto the upper half-plane $\mathbb{H}$. Since the Green's function is conformally invariant and the Green's function of $\mathbb{H}$ with the pole at $w_{0} \in \mathbb{H}$ is given by $g_{\mathbb{H}}\left(w, w_{0}\right)=\log \left|\frac{w-\bar{w}_{0}}{w-w_{0}}\right|$, we have

$$
g_{\Omega(Q(d, 1))}(0, \infty)=\log \left|\frac{f(0)-\overline{f(\infty)}}{f(0)-f(\infty)}\right|=\log \frac{\sin \frac{1}{3}(\pi+2 \operatorname{arccot} d)}{\sin \frac{1}{3}(\pi-2 \operatorname{arccot} d)} .
$$

In the second limit case, when $r=(1 / 2) \sqrt{4-d^{2}}$, the conformal mapping from the upper half-plane onto $\Omega(Q(d))$, and therefore the Green's function of the complement of the circular triangle $Q(d)$, can be found in the closed form (see [2, Section V.7]), but this form will contain parameters defined implicitly by highly transcendental equations.

It is interesting to compare $g_{\Omega(Q(d, 1))}(0, \infty)$ with the Green's function of the complement of an arc of the unit circle subtended by a chord of length $d$. Let $C(d)=\left\{e^{i \theta}: \cos \theta \leq-(1 / 2) \sqrt{4-d^{2}}\right\}$. Then straightforward calculations left to the interested reader show that

$$
g_{\Omega(C(d))}(0, \infty)=\log 2-(1 / 2) \log \left(2-\sqrt{4-d^{2}}\right) .
$$

Using the explicit expressions in (19) and (20) and the fact that $C(d) \subset Q(d)$, it is not difficult to show that

$$
g_{\Omega(Q(d))}(0, \infty)<g_{\Omega(C(D))}(0, \infty)<g_{\Omega(Q(d, 1))}(0, \infty)
$$

for values of $d$ sufficiently close to 2 . The latter inequality provides some numerical support to our Conjecture 2

\section{ACKNOWLEDGEMENT}

The author would like to acknowledge that Proof 1 of the main theorem was suggested by the referee. The author wants to thank the referee for this and other valuable suggestions. 


\section{REFERENCES}

[1] N. A. Lebedev, Printsip ploshchadei v teorii odnolistnykh funktsii (Russian), Izdat. "Nauka", Moscow, 1975. MR.0450540 (56 \#8834)

[2] Zeev Nehari, Conformal mapping, Dover Publications Inc., New York, 1975. Reprinting of the 1952 edition. MR0377031 (51 \#13206)

[3] George Pólya and Gabor Szegő, Problems and theorems in analysis. II, Theory of functions, zeros, polynomials, determinants, number theory, geometry; translated from the German by C. E. Billigheimer; reprint of the 1976 English translation. Classics in Mathematics, SpringerVerlag, Berlin, 1998. MR1492448

[4] A. Yu. Solynin, Polarization and functional inequalities (Russian, with Russian summary), Algebra i Analiz 8 (1996), no. 6, 148-185; English transl., St. Petersburg Math. J. 8 (1997), no. 6, 1015-1038. MR.1458141 (98e:30001a)

[5] P. M. Tamrazov, Extremal conformal mappings and poles of quadratic differentials (Russian), Izv. Akad. Nauk SSSR Ser. Mat. 32 (1968), 1033-1043. MR0235105 (38 \#3417)

[6] Vilmos Totik, Christoffel functions on curves and domains, Trans. Amer. Math. Soc. 362 (2010), no. 4, 2053-2087, DOI 10.1090/S0002-9947-09-05059-4. MR2574887 (2011b:30006)

Department of Mathematics and Statistics, Texas Tech University, Box 41042, LuBBOCK, TEXAS 79409

E-mail address: alex.solynin@ttu.edu 